\title{
THE STRUCTURE OF A CLASS OF REPRESENTATIONS OF THE UNITARY GROUP ON A HILBERT SPACE
}

\author{
I. E. SEGAL ${ }^{1}$
}

1. In the mathematical theory of fields of elementary particles one has to do with a representation of the unitary group on a Hilbert space $H$. With any such representation $\Gamma$ and subspace $M$ of $H$ there is associated a self-adjoint operator called the "number of particles in $M . "$ The particle interpretation of general wave fields is made possible in the first instance by the fact that these operators have integral proper values. The direct physical approach suggests that these proper values should be non-negative, which however is not the case for an arbitrary representation. The purpose of the present note is to determine the structure of the most general "physical" representation, i.e., one for which the number of particles is always non-negative. It is shown that this structure is essentially the same as in the case of a finite-dimensional Hilbert space.

Specifically, an irreducible physical representation is unitarily equivalent to the canonical representation in a class of covariant tensors of maximal symmetry over the "one-particle" space $H$. The most general physical representation is a direct sum of these irreducible covariant tensor representations. For a finite-dimensional space, these results are essentially equivalent to well-known ones giving the structure of the general unitary representation of the unitary group on the space. These known results are established by the use of characters, a technique which is not adaptable to the infinite-dimensional case because there is then no trace for unitary operators on the space. The method employed is rather to approximate the space by subspaces of high finite dimension and to make use of what is already known in the finite-dimensional case.

2. Throughout this paper, $G$ will designate the group of unitary operators on a complex Hilbert space $H$. Unless otherwise specified, the dimension of $H$ may be arbitrary. $\Gamma$ will denote a given continuous unitary representation of $G$ on a complex Hilbert space $K$. The topology of $G$ that is used is the so-called "strong" operator topology. For any self-adjoint operator $A$ on $H$, the self-adjoint generator of the one-parameter group $\left\{\Gamma\left(e^{i t A}\right):-\infty<t<\infty\right\}$ will be designated $d \Gamma(A)$ and the mapping $d \Gamma$ from the self-adjoint operators in $H$ into those

Received by the editors September 5, 1955 and, in revised form, January 31, 1956.

1 Research supported in part by a contract with the Office of Naval Research. 
in $K$ will be called the infinitesimal representation associated with $\Gamma$. If $P$ is a projection with range $M, d \Gamma(P)$ is called the number of particles in $M$. The representation $\Gamma$ is called "physical" in case these operators are non-negative.

Theorem. A continuous physical unitary representation of the unitary group on a complex Hilbert space is a direct sum of irreducible such representations. The latter are unitarily equivalent to the canonical representations of the group on the spaces of covariant tensors of maximal symmetry types.

3. We recall that the space $H_{n}$ of covariant tensors over $H$ of rank $n$ is itself a Hilbert space, which is spanned by products of the form $x_{1} \otimes x_{2} \otimes \cdots \otimes x_{n}$, where $x_{i}$ range over an arbitrary basis for $H$, and in which the inner product is determined by the rule that

$$
\left(x_{1} \otimes x_{2} \otimes \cdots \otimes x_{n}, y_{1} \otimes \cdots \otimes y_{n}\right)=\prod_{i=1}^{n}\left(x_{i}, y_{i}\right) .
$$

For any unitary operator $U$ on $H$, there is a unique unitary operator $\Gamma_{n}(U)$ on $H_{n}$ taking $x_{1} \otimes \cdots \otimes x_{n}$ into $U x_{1} \otimes \cdots \otimes U x_{n}$. For any element $\pi$ of the symmetric group $\sum_{n}$ on the first $n$ positive integers there is a unique unitary operator $V_{n}(\pi)$ on $H_{n}$ taking $x_{1} \otimes \cdots \otimes x_{n}$ into $x_{\pi(1)} \otimes \cdots \otimes x_{\pi(n)}$. A symmetry "type" is a projection $Q_{n}$ in the algebra $A\left(\sum_{n}\right)$ of $V_{n}$, a tensor in the range of $Q_{n}$ being said to be of symmetry type $Q_{n}$. The ordering on symmetry types is the reverse of the ordering on the corresponding projections, so that the range $L_{n}$ of a minimal projection in $A\left(\sum_{n}\right)$ is a space of covariant tensors of maximal symmetry type. The $\Gamma_{n}(U)$ and the $V_{n}(\pi)$ commute, so that $L_{n}$ is invariant under the $\Gamma_{n}(U)$. The mapping

$$
U \rightarrow \Gamma_{n}(U) \mid L_{n}
$$

is a continuous unitary representation, and is known to be irreducible. It is not difficult to verify that it is physical. For further details and references, see [1].

At this point we collect some known facts about the representations of the unitary group on a finite-dimensional space that will be used in the proof of the theorem. The general irreducible representation $U$ of the group $G_{d}$ on a $d$-dimensional space may be specified by a $d$-tuple $\left(n_{1}, n_{2}, \cdots, n_{d}\right)$ of integers in which $n_{1} \geqq n_{2} \geqq \cdots \geqq n_{d}$. When the $n_{k}$ are all non-negative, the positive ones give a partition of the sum $n$ for which a corresponding minimal projection in the algebra of $\sum_{n}$ determines a symmetry class of covariant tensors of rank $n$ on which the associated action of the unitary group is equiva- 
lent to $U$. The general case is reducible to this one through the fact that in general the representation of type $\left(n_{1}+p, n_{2}+p, \cdots, n_{d}+p\right)$ is equivalent to the direct product of the representation of type $\left(n_{1}, n_{2}, \cdots, n_{d}\right)$ with the representation $a \rightarrow(\operatorname{det} a)^{p}$. The irreducible representation of $G_{d+1}$ of type $\left(n_{1}, n_{2}, \cdots, n_{d+1}\right)$ splits on restriction to the subgroup leaving one nonzero vector invariant, and hence isomorphic to $G_{d}$, into a direct sum of representations of $G_{d}$ of type $\left(n_{1}^{\prime}, \cdots, n_{d}^{\prime}\right)$, where $n_{1} \geqq n_{1}^{\prime} \geqq n_{2} \geqq \cdots \geqq n_{d}^{\prime} \geqq n_{d+1}$, each such representation occurring exactly once. For these and related facts about the representations of the finite-dimensional unitary group, see [2] and $[\mathbf{3}]$.

4. We need a fact mentioned above, namely

Lemma 1. For any continuous unitary representation of the unitary group on a Hilbert space, the number of particles in any subspace has integral proper values. ${ }^{2}$

If $P$ is the projection on the given subspace, then the map $t \rightarrow e^{i t P}$, $0 \leqq t<2 \pi$, gives a continuous unitary representation of the reals modulo $2 \pi$. Hence the same is true of the map $t \rightarrow \Gamma\left(e^{i t P}\right), \Gamma$ being the given representation. Now any continuous unitary representation of the reals modulo $2 \pi$ is a direct sum of the character representations $t \rightarrow e^{i n t}$, with $n=0, \pm 1, \cdots$. On a subspace on which $\Gamma\left(e^{i t P}\right)$ acts as multiplication by $e^{i n t}, d \Gamma(P)$ acts as multiplication by $n$ and the lemma follows.

In particular the total number of particles $d \Gamma(I)$, where $I$ denotes the identity operator on $H$, has integral proper values. As the unitary group $\left\{e^{i t I} ; 0 \leqq t<2 \pi\right\}$ commutes with every unitary operator $U$ on $H$, the $\Gamma\left(e^{i t I}\right)$ commutes with all $\Gamma(U)$. From this it follows readily that the $\Gamma(U)$ leave invariant the spectral manifolds of $d \Gamma(I)$. It follows that it is no essential loss of generality to assume that the total number of particles has a fixed non-negative integral value $n$ (more precisely is $n$ times the identity).

Making this assumption, we note that the number of particles in any subspace is always at most $n$. For if $P$ is the projection on the subspace, then from the fact that $e^{i t P}$ and $e^{i s(I-P)}$ commute for $-\infty<s, t<\infty$, it results that $d \Gamma(P)$ and $d \Gamma(I-P)$ commute in the sense that they have a simultaneous spectral resolution. It follows that $d \Gamma(I)$ is the closure of the sum $d \Gamma(P)+d \Gamma(I-P)$. As $d \Gamma(I)$ is a scalar multiple of the identity on the representation space, it results that $d \Gamma(P)$ is bounded and in fact $d \Gamma(P) \leqq d \Gamma(I)$.

${ }^{2}$ This lemma and its proof are valid without the assumption made in the remainder of the paper that the representation is physical. 
5. For any finite-dimensional subspace $M$ of $H$, let $G_{M}$ be the subgroup of $G$ leaving vectors in the orthocomplement of $M$ invariant. Then $G_{Y I}$ is isomorphic to the unitary group on $M$ in a canonical and obvious fashion, and the two groups will be identified where the context makes the meaning clear, in order to avoid uncalled-for verbiage. Putting $\Gamma_{M}$ for the restriction of $\Gamma$ to $G_{M}$ and $P_{M}$ for the projection with range $M, d \Gamma\left(P_{M}\right)$ may be identified with $d \Gamma_{M}\left(I_{M}\right)$, where $I_{M}$ denotes the identity operator on $M$. As $d \Gamma\left(P_{M}\right) \leqq d \Gamma(I)$, it results that $d \Gamma_{M}\left(I_{M}\right)$ is bounded by $n$. Now $\Gamma_{M}$ is a direct sum of irreducible covariant tensor representations of types $\left(n_{1}, n_{2}, \cdots, n_{d}\right)$, where $d$ is the dimension of $M$. It is easily verified that the total number of particles for a representation of this type is $\sum_{j} n_{j}$. It follows that only the types with

can arise.

$$
n_{1}+n_{2}+\cdots+n_{d} \leqq n
$$

A further restriction on the types that may arise in this fashion is that all the $n_{i}$ must be non-negative. For suppose that the type $\left(n_{1}, n_{2}, \cdots, n_{d}\right)$ occurs with $n_{d}=-h$ and $h>0$. Let $\left\{x_{1}, x_{2}, \cdots, x_{d}\right\}$ be an arbitrary orthonormal basis for $M$, and let $Q$ be the projection on the one-dimensional subspace spanned by $x_{d}$. On the one hand, $d \Gamma(Q) \geqq 0$ since $\Gamma$ is assumed to be physical. On the other hand, consider the action of $d \Gamma(Q)$ on the tensor $w$ obtained by antisymmetrization of $x_{1} \otimes \cdots \otimes x_{1} \otimes x_{2} \otimes \cdots \otimes x_{2} \otimes \cdots \otimes x_{d-1}$, where each $x_{j}$ occurs $n_{j}$ times $(j<d)$, regarding $\Gamma$ as the direct product of the representation of type $\left(n_{1}+h, n_{2}+h, \cdots, n_{d-1}+h, 0\right)$ with the representation $a \rightarrow(\operatorname{det} a)^{h}$. It is readily computed that $d \Gamma(Q) w$ $=-h w$, where the tensor $w$ is identified with the corresponding element of the representation space of $\Gamma$. This implies $w=0$, which can not be the case for all such $w$ as the known form of the representations of the symmetric group shows that all such w span the range of a nonvanishing class of tensors of maximal symmetry type.

6. Let two types $\left(n_{1}, n_{2}, \cdots, n_{d}\right)$ and $\left(n_{1}^{\prime}, n_{2}^{\prime}, \cdots, n_{d}^{\prime}\right)$ be called "essentially equivalent" in case the $n_{j}$ are the same as the $n_{j}^{\prime}$ when zero elements are disregarded. That is, $n_{j}=n_{j}^{\prime}$ for $j \leqq \min \left(d, d^{\prime}\right)$. It is clear from the preceding two sections that only a finite number of essentially inequivalent types occur in the reduction of the various $\Gamma \mid G_{M}$, as $M$ ranges over the finite-dimensional subspaces $H$. It follows that there exists a maximal type $\left(\bar{n}_{1}, \bar{n}_{2}, \cdots, \bar{n}_{\bar{d}}\right)$, in the sense that if a type $\left(n_{1}, \cdots, n_{d}\right)$ occurs with $n_{j} \geqq \bar{n}_{j}$, and $d \geqq \bar{d}$, then either $n_{j}=\bar{n}_{j}$ or $j>\bar{d}$ and $n_{j}=0$.

Let $M$ be a fixed finite-dimensional subspace of dimension $m$, such 
that the maximal type occurs in the reduction of $\Gamma \mid G_{M}$. Let $K_{M}$ be a subspace of the representation space $K$ such that the contractions of the $\Gamma(U), U \in G_{M}$, to $K_{M}$ give a representation of $G_{M}$ of this type. If $N$ is any finite-dimensional subspace containing $M$, there is a minimal subspace $K_{N}$ of $K$ that contains $K_{M}$ and is invariant under $\Gamma\left(G_{N}\right)$. The next step in the proof is to show that $K_{N}$ is automatically minimal with respect to being invariant under $\Gamma\left(G_{N}\right)$, and that the contraction of $\Gamma_{N}$ to $K_{N}$ has the maximal type.

7. Let $\left\{e_{1}, e_{2}, \cdots, e_{n}\right\}$ be an orthonormal basis for $N$ such that $\left\{e_{1}, e_{2}, \cdots, e_{m}\right\}$ is an orthonormal basis for $M$. Now $N$ can be obtained from $M$ by successive adjunction of $e_{m+1}, e_{m+2}, \cdots, e_{n}$. It is not difficult to see from this that if the conclusion cited at the end of the preceding section is valid when $n=m+1$, then it holds in general. We now assume this to be the case, that $n=m+1$.

By the branching law cited above, an irreducible representation of $G_{N}$ of type $\left(p_{1}, p_{2}, \cdots, p_{n}\right)$ branches, on restriction to $G_{M}$, into a representation equivalent to a direct sum of the (inequivalent) representations of the types $\left(p_{1}^{\prime}, p_{2}^{\prime}, \cdots, p_{n-1}^{\prime}\right)$, where

$$
p_{1} \geqq p_{1}^{\prime} \geqq p_{2} \geqq p_{2}^{\prime} \geqq \cdots \geqq p_{m}^{\prime} \geqq p_{n} .
$$

By the orthogonality of inequivalent irreducibly invariant subspaces of a compact group, $K_{M}$ is orthogonal to the representation space of any such representation of $G_{N}$ unless

$$
p_{1} \geqq n_{1} \geqq p_{2} \geqq n_{2} \geqq \cdots \geqq n_{m} \geqq p_{n} .
$$

That is, $K_{M}$ is contained in the direct sum of subspaces that are irreducibly invariant under $\Gamma\left(G_{N}\right)$ and on which the action of $G_{N}$ is of the maximal type.

It follows that to show the irreducibility of $K_{N}$ it suffices to establish

Lemma 2. Let the unitary representation $V$ of the group $R$ be a multiple of the irreducible representation $V_{1}$. Let $S$ be a subgroup of $R$ such that the restriction of $V_{1}$ to $S$ splits into a direct sum of inequivalent representations. Then any subspace irreducibly invariant under the restriction of $V$ to $S$ is contained in a subspace irreducibly invariant under $V$.

To prove the lemma, we may take the representation space to have the form $E_{1} \otimes E_{2}$, and $V$ to have the form $V(a)=V_{1}(a) \otimes I_{2}, a \in R$. Then the ring $A_{1}$ generated by the $V_{1}(a)$, for $a \in R$, is the ring of all bounded operators on $E_{1}$, while the ring $B$ generated by the $V_{1}(a)$, $a \in S$, has by virtue of the branching assumption the property that its 
commutor $B^{\prime}$ is abelian. (Here the term "ring" is used in its Hilbert space sense, as a weakly closed self-adjoint algebra of bounded operators including the identity operator.) Now the projection $P$ on any subspace irreducibly invariant under the restriction of $V$ to $S$ is minimal in the commutor of the ring generated by the $V_{1}(a) \otimes I_{2}$, $a \in S$, which commutor is seen to be $B^{\prime} \otimes A_{2}$, where $A_{2}$ is the ring of all bounded operators on $H_{2}$.

It follows readily from the abelian character of $B^{\prime}$ that any such projection has the form $P_{1} \otimes P_{2}$ with $P_{1}$ minimal in $B^{\prime}$ and $P_{2}$ minimal in $A_{2}$. Now $I_{1} \otimes P_{2}$ is minimal in the commutor of the ring $A_{1} \otimes J_{2}$ generated by the $V(a)$ for $a \in R$, where $J_{i}$ is the ring of all scalar multiples of $I_{i}$, since this commutor is $J_{1} \otimes A_{2}$. As it is obvious that $I_{1} \otimes P_{2} \geqq P_{1} \otimes P_{2}$ the lemma is proved.

COMPLETION OF PROOF. It has now been shown that there exists a finite-dimensional subspace $M$ of $H$, and irreducible representation type $\left(f_{1}, f_{2}, \cdots, f_{n}\right), f_{1} \geqq f_{2} \geqq \cdots \geqq f_{n}>0$, and an irreducibly invariant subspace $K_{M}$ under $\Gamma\left(G_{M}\right)$, on which the action of $G_{M}$ is of this type, and such that whenever $N$ is a finite-dimensional subspace containing $M$, there is an irreducibly invariant subspace $K_{N}$ under $\Gamma\left(G_{N}\right)$ that contains $K_{M}$, and on which the action of $G_{N}$ is of type $\left(f_{1}, f_{2}, \cdots, f_{n}, 0,0, \cdots, 0\right)$. The union $L$ of the $K_{N}$ is invariant under any $\Gamma\left(G_{N}\right)$ for $N$ finite-dimensional and, as the $G_{N}$ generate $G$ in the strong topology, is invariant under $\Gamma(G)$.

Now let $\Gamma^{\prime}$ on $K^{\prime}$ be the tensor representation of $G$ of type $\left(f_{1}, f_{2}, \cdots, f_{r}\right)$. To conclude the proof it suffices to show that $\Gamma^{\prime}$ is unitarily equivalent to the representation $\Gamma^{L}: U \rightarrow \Gamma(U) \mid L$ obtained by restricting all the operators $\Gamma(U)$ to $L$. For as shown in [1], $\Gamma^{\prime}$ is irreducible, so that it would follow that every continuous physical unitary representation contains an irreducible such representation, and hence is a direct sum of them. It would further follow that the most general irreducible physical representation is as stated.

To set up the unitary equivalence between $\Gamma^{L}$ and $\Gamma^{\prime}$ let $x$ be an element of a $K_{N}$ for some finite-dimensional subspace $N$ containing $M$. Now the restriction of $\Gamma$ to $G_{N}$ acts on $K_{N}$ in a fashion unitarily equivalent to the representation of $G_{N}$ of type $\left(f_{1}, f_{2}, \cdots, f_{r}\right)$. Hence there exists a unitary transformation, say $W_{N}$, from $K_{N}$, onto the subspace $K_{N}^{\prime}$ of $K^{\prime}$ consisting of those tensors that are in the tensorsubspace spanned by direct products of elements of $N$, and of symmetry type $\left(f_{1}, \cdots, f_{r}\right)$, that exchanges the contraction to $G_{N}$ of $\Gamma^{K_{N}}$ with $\Gamma^{\prime} \mid G_{N}$. For it is clear from the form stated above for the representations of type $\left(f_{1}, \cdots, f_{r}\right)$ that the contraction of $\Gamma^{\prime}$ to $G_{N}$ leaves invariant the stated tensor subspaces and acts on it ir- 
reducibly of type $\left(f_{1}, \cdots, f_{r}\right)$. By virtue of the irreducibility of $K_{N}$ under $\Gamma\left(G_{N}\right), W_{N}$ is unique within a multiplication by a scalar of absolute value one.

Now by definition of $W_{N}$, it takes $K_{N}$ onto $K_{N}^{\prime}$ in such a fashion that

$$
W_{N} \Gamma(U) W_{N}^{-1}=\Gamma^{\prime}(U) \mid K_{N}^{\prime}, \quad U \in G_{N},
$$

as the $\Gamma^{\prime}(U), U \in G_{M}$, leave $W_{N} K_{M}^{\prime}$ invariant and give a representation of type $\left(f_{1}, \cdots, f_{r}\right)$ of $G_{M}$. Now the branching law shows that $K_{N}$ contains a unique irreducibly invariant subspace of type $\left(f_{1}, \cdots, f_{n}\right)$ under $\Gamma\left(G_{M}\right)$, and hence $W_{N} K_{M}^{\prime}=K_{M}$. Restricting the defining relation for $W_{N}$ to the elements of $G_{M}$, it follows that

$$
W_{M} \Gamma(U) W_{M}^{-1}=W_{N} \Gamma(U) W_{M}^{-1} \mid K_{M}^{\prime}
$$

for $U \in G_{M}$. It results that $W_{M}=\alpha_{N}\left(W_{N} \mid K_{M}^{\prime}\right)$, where $\alpha_{N}$ is of absolute value one. Now choosing $W_{M}$ in an arbitrary fashion and choosing $W_{N}$ so that $\alpha_{N}=1$, the $W_{N}$ are uniquely defined relative to the choice of $W_{M}$.

Moreover, by the argument just employed, whenever $N^{\prime} \supset N, W_{N^{\prime}}$ extends $W_{N}$. It is easy to deduce the existence of a unique unitary transformation $W$ from $L$ onto $K^{\prime}$ that extends all the $W_{N}$. This unitary $W$, by virtue of the fact that it agrees with $W_{N}$ on $K_{N}$, has the property

$$
W \Gamma(U) W^{-1}=\Gamma^{\prime}(U) \mid K_{N}^{\prime}, \quad U \in G_{N}^{\prime},
$$

for each $N$. Now if $N \subset N^{\prime}, G_{N} \subset G_{N^{\prime}}$, so that

$$
W \Gamma(U) W^{-1}=\Gamma^{\prime}(U) \mid K_{N^{\prime}}^{\prime}, \quad U \in G_{N} .
$$

Passing to the limit with the family $\left\{N^{\prime}\right\}$, directed by inclusion, it results that

$$
W \Gamma(U) W^{-1}=\Gamma^{\prime}(U), \quad U \in G_{N} .
$$

Making a similar second passage to the limit shows the stated unitary equivalence.

\section{REFERENCES}

1. I. E. Segal, Tensor algebras over Hilbert spaces. I, Trans. Amer. Math. Soc. vol. 81 (1956) pp. 106-134.

2. H. Weyl, The classical groups, Princeton, 1939.

3. - Gruppentheorie und Quantenmechanik, 2d ed., Leipzig, 1931.

University of Chicago 